\title{
Urinary Metabolites and Their Link with Premature Arterial Stiffness in Black Boys: The ASOS Study
}

\author{
Dalene Erasmus $^{a} \quad$ Carina M.C. Mels ${ }^{a}, b \quad$ Roan Louw $^{c} \quad$ J. Zander Lindeque ${ }^{c}$ \\ Ruan Kruger ${ }^{a, b}$ \\ ${ }^{a}$ Hypertension in Africa Research Team (HART), North-West University - Potchefstroom \\ Campus, Potchefstroom, South Africa; ${ }^{b}$ MRC Research Unit for Hypertension and \\ Cardiovascular Disease, North-West University, Potchefstroom, South Africa; \\ 'Human Metabolomics, North-West University - Potchefstroom Campus, Potchefstroom, \\ South Africa
}

\section{Keywords}

$\beta$-Alanine $\cdot$ Cardiac protection $\cdot$ L-Proline $\cdot$ Metabolomics $\cdot$ Pulse wave velocity

\begin{abstract}
Background and Aims: Black boys (6-8 years of age) were shown to have higher pulse wave velocity with potential early vascular compromise. We aimed to compare predefined urinary metabolites in black and white boys to explore associations of pulse wave velocity with these metabolites. Methods and Results: We included 40 white and 40 black apparently healthy boys between the ages of 6 and 8 years. Femoral pulse wave velocity was measured along with various metabolites using liquid chromatography tandem mass spectrometry (LC-MS/ MS) and gas chromatography-time of flight-mass spectrometry (GC-TOF-MS) methods. Pulse wave velocity and diastolic blood pressure were higher in the black compared to the white boys (both $p \leq 0.002$ ). Isovalerylcarnitine was lower and 1-metylhistidine tended to be lower $(p=0.002$ and $p=0.073$, respectively), whereas L-proline levels tended to be higher $(p=0.079)$ in the black compared to the white boys. In single, partial, and multiple regression analyses, pulse wave velocity correlated inversely with $\beta$-alanine $(\beta=-0.414 ; p=0.008)$ and 1 -methylhistidine $(\beta=-0.347 ; p=0.032)$ and positively with L-proline $(\beta=0.420 ; p=0.008)$, threonic $\operatorname{acid}(\beta=0.977 ; p=0.033)$, and malonic acid $(\beta=0.348 ; p=0.030)$ in black boys only. Conclusion: Our study is the first to discover the associations of pulse wave velocity with $\beta$-alanine, 1-methylhistidine, and L-proline in children from South Africa, which may suggest potential early compromise in cardiac protective metabolic pathways in black boys as young as 6 years of age.


Erasmus et al.: Metabolomics and Arterial Stiffness in Boys

\section{Introduction}

In recent years, the prevention of cardiovascular disease (CVD) has received a great deal of attention through the identification of cardiac protective targets [1-3]. A recent South African study showed black boys (aged 6-8 years) to have increased pulse wave velocity, intima-media thickness, advanced glycation products, and diastolic blood pressure [4]. Lower arterial compliance in black boys (aged 10-15 years) compared to their white counterparts was also previously reported [5]. These findings highlighted the importance of investigating the molecular basis of these early changes, to develop potential interventions and identify cardiac protective targets to prevent the early onset of CVD [6].

In the quest to identify new targets for cardiac protection and ultimately prevent or delay the onset of CVD, hypothesis-generating techniques such as metabolomics are frequently employed. Metabolomics focus on the integrated recognition of biological and pathological pathways, providing the ability to reflect the physiological state of the body [7, 8]. Targeted metabolomics make use of extreme selective and precise analytical platforms to perform quantitative measuring of a specific list of metabolites, which may include amino acids, acylcarnitines, and organic acids [8]. With targeted metabolomics, the chemical composition of the metabolites is known before the data are obtained, with fewer data detected, whereas untargeted metabolomics is the measuring of metabolites where thousands of metabolites can be detected without prior knowledge of the chemical composition [9]. Analytical techniques such as liquid chromatography tandem mass spectrometry (LC-MS/MS) and gas chromatography-time of flight-mass spectrometry (GC-TOF-MS) methods are frequently used in metabolomics studies $[8,10]$.

To the best of our knowledge, targeted and untargeted metabolomics studies along with the identification of specific metabolites related to arterial stiffness in children are sparse. We therefore aimed to explore associations of pulse wave velocity as a measure of arterial stiffness with targeted and untargeted metabolites to identify which metabolites to focus on in this study. The identified urinary metabolites were compared between black and white boys and independent associations of pulse wave velocity with these metabolites were explored.

\section{Methods of Data Collection}

\section{Population Description and Sample Size}

The Arterial Stiffness in Offspring Study (ASOS) was conducted in 2015 and included 81 (41 white and 40 black) apparently healthy boys (with no known [self-reported by parents] chronic or infectious illnesses) between the specific ages of 6 and 8 years. All the boys were recruited from the same schools in Potchefstroom in the North-West province, South-Africa. Only boys with participation permission letters signed by their parents were included in this study. The exclusion criteria were as follows: girls, boys with self-reported (by the parents) type I diabetes mellitus, renal disease, or cancer, as well as obese boys and those using chronic medication. In the present study, 1 participant with missing metabolomics data was excluded, hence we used a sample size of 80 participants ( 40 white and 40 black boys).

This study conformed to the Declaration of Helsinki and was approved by the Health Research Ethics Committee of the North-West University (NWU-00049-17-A1). The screening of the participants started at 08:00 in the morning after receiving all the relevant questionnaires, consent forms signed by boys older than 7 years, and assent forms signed by children younger than 7 years and their parents. Anthropometric and cardiovascular measurements were taken, and each child received refreshments when these measurements were completed. 


\section{Anthropometric Measurements}

Body height $(\mathrm{cm})$ and weight $(\mathrm{kg})$ were measured with a Seca 213 stadiometer and a Seca 813 digital scale, respectively (Birmingham, UK). Subsequently, body mass index was calculated for each child and recorded to the dataset for further analysis according to the World Health Organization (WHO) classification (z-scores) of paediatric anthropometry (Birmingham, UK). By using a Lufkin ${ }^{\circledR}$ Executive Thinline 2-mm steel tape (Apex Tool Group B.V.; Emmen, The Netherlands), waist circumferences were measured in triplicate according to standard procedures [11].

\section{Cardiovascular Measurements}

Office blood pressure and heart rate was measured for each participant in a rested, sitting position using a paediatric validated Omron HEM-759-E (705IT) device (Omron Healthcare, Tokyo, Japan). Femoral pulse wave velocity was measured with the Complior SP Acquisition System (Artech Medical, Pantin, France). A Lufkin ${ }^{\circledR}$ Executive Thinline 2-mm steel tape (Apex Tool Group B.V.) was used to calculate the distances between the pulsated sections, where $80 \%$ was used as the pulse wave travelled distance [12].

\section{Biochemical Analysis}

All participants were requested to provide a first voided midstream morning urine sample before eating or drinking anything. The urine sample of each participant was received at the school office and taken to the research laboratory where it was prepared and stored at $-80^{\circ} \mathrm{C}$. Urinary creatinine levels were measured using the Cobras Integra ${ }^{\circledR} 400$ plus (Roche Diagnostics Mannheim, Germany). All metabolomics measurements were then normalized to creatinine levels.

Amino acids and acylcarnitines were analysed with the LC-MS/MS method. A specific volume of each urine sample, containing $0.0625 \mu \mathrm{mol}$ of creatinine, was transferred to a glass vial and $1 \mathrm{~mL}$ of deuterated isotope mixture used as internal standard was added. The deuterated isotope mixture contained valine (70.5 ppm), isoleucine (22.0 ppm), phenylalanine (57 ppm), lysine (57.5 ppm), free L-carnitine (73.5 ppm), acetylcarnitine (137 ppm), octanoylcarnitine $(77.5 \mathrm{ppm})$, and octadecanoylcarnitine (59 ppm) isotopes. The samples were then evaporated to dryness under nitrogen gas $\left(\mathrm{N}_{2}\right)$ at $37^{\circ} \mathrm{C}$. Then, $200 \mu \mathrm{L}$ of $3 \mathrm{~N}$ butanolic hydrochloric acid was added to the dried residue, and the samples were incubated at $50^{\circ} \mathrm{C}$ for $60 \mathrm{~min}$. The butylated samples were again evaporated to dryness with $\mathrm{N}_{2}$ at $37^{\circ} \mathrm{C}$. The dried residue was reconstituted in $100 \mu \mathrm{L}$ water:acetonitrile (50:50 v/v) containing $0.1 \%$ formic acid. The samples were analysed on an Agilent 6410 LC-MS/MS system with 1,200 series liquid chromatograph front end. Separation was done with a C18 Zorbax SB-Aq (150 mm $\times 2.1 \mathrm{~mm} \times 3.5 \mu \mathrm{m})$ column from Agilent. The column was kept at $30{ }^{\circ} \mathrm{C}$ during the entire run. The sample injection volume was $1 \mu \mathrm{L}$. The chromatographic gradient started at $95 \%$ solvent A (water with $0.1 \%$ formic acid) with a flow rate of $0.2 \mathrm{~mL} / \mathrm{min}$ maintained for $1 \mathrm{~min}$ before the gradient was increased to $18 \%$ solvent B (acetonitrile with $0.1 \%$ formic acid) over a period of $4 \mathrm{~min}$. The gradient was then kept constant for $3 \mathrm{~min}$. Next, the gradient was increased linearly to $100 \%$ solvent B for $15 \mathrm{~min}$. Over this period, the flow was linearly increased to $0.3 \mathrm{~mL} / \mathrm{min}$. After maintaining the flow at $0.3 \mathrm{~mL} / \mathrm{min}$ for $5 \mathrm{~min}$ with $100 \%$ solvent B, the flow and gradient was gradually decreased to $0.2 \mathrm{~mL} / \mathrm{min}$ and $5 \%$ solvent B over a 3-min period. A post-run of 10 min was allowed to ensure equilibration of the column to give a total run time of $33 \mathrm{~min}$ per sample (23 min gradient and $10 \mathrm{~min}$ post-run). The electrospray ionization source gas temperature was kept at $300^{\circ} \mathrm{C}$, with a flow rate of $7.5 \mathrm{~L} / \mathrm{min}$. Nebulizer pressure was kept at 30 psi and capillary voltage at 3,500 V. LC-MS/MS data were processed with MassHunter Quantitative Analysis software according to the vendor's specification.

GC-TOF-MS analyses was performed to obtain relative quantities of metabolites. The volume of urine samples used was calculated from the creatinine concentration to compensate for variation in urine concentrations. The calculated volume of urine containing $0.125 \mu \mathrm{mol}$ of creatinine and internal standard $(100 \mathrm{ppm}$ 3-phenylbutyric acid) was transferred to a glass vial and dried under nitrogen gas for 45 min at $37^{\circ} \mathrm{C}$. Derivatization (oximation and silylation) of samples to achieve sufficient volatility for gas chromatography was performed. Oximation was performed by adding $50 \mu \mathrm{L}$ of oximation reagent $(2 \mathrm{mg} / \mathrm{mL}$ methoxyamine in pyridine) to the dried samples and followed by incubation for $60 \mathrm{~min}$ at $60^{\circ} \mathrm{C}$. Silylation followed with the addition of $50 \mu \mathrm{L}$ of $\mathrm{N}, \mathrm{O}$-Bis (trimethylsilyl) trifluoroacetamide (containing $1 \%$ trimethylsilyl chloride), after which the samples were vortexed for $1 \mathrm{~min}$ and incubated for $60 \mathrm{~min}$ at $60^{\circ} \mathrm{C}$. The samples were then analysed on a Leco Pegasus HT GC-TOF-MS system with Agilent 7890A gas chromatography front end. Separation was done with a Rxi-5Sil MS (30 m $\times 250 \mu \mathrm{m} \times 0.25 \mu \mathrm{m})$ column from Restek. Helium was used as carrier gas at a constant flow rate of $1.5 \mathrm{~mL} / \mathrm{min}$. The inlet temperature was kept at $250^{\circ} \mathrm{C}$ for injection. The sample injection volume was $1 \mu \mathrm{L}$ with $1: 10$ split. The oven temperature was initiated at $70^{\circ} \mathrm{C}$ for $1 \mathrm{~min}$ 
Erasmus et al.: Metabolomics and Arterial Stiffness in Boys

Table 1. Characteristics of the study population

\begin{tabular}{lllc}
\hline & $\begin{array}{l}\text { Black children } \\
(n=40)\end{array}$ & $\begin{array}{l}\text { White children } \\
(n=40)\end{array}$ & $p$ value \\
\hline Age, years & $7.80 \pm 0.73$ & $7.68 \pm 0.98$ & 0.52 \\
Waist circumference, cm & $56.9 \pm 5.67$ & $58.0 \pm 5.76$ & 0.34 \\
Height, cm & $125 \pm 6.37$ & $126 \pm 7.18$ & 0.87 \\
Waist-to-height ratio & $0.83 \pm 0.03$ & $0.87 \pm 0.04$ & $\leq 0.001$ \\
Body mass index & $16.5 \pm 2.08$ & $16.0 \pm 1.71$ & 0.34 \\
\hline Cardiovascular measurements & & & 0.16 \\
Systolic blood pressure, mm Hg & $105 \pm 10.9$ & $102 \pm 7.13$ & $\leq 0.001$ \\
Diastolic blood pressure, mm Hg & $69.4 \pm 8.96$ & $62.6 \pm 7.64$ & $\leq .002$ \\
Mean arterial pressure, mm Hg & $81.4 \pm 9.01$ & $75.9 \pm 6.70$ & $\leq 0.001$ \\
Pulse wave velocity, $\mathrm{m} / \mathrm{s}^{*}$ & $5.13(4.88 ; 5.39)$ & & $0.37(4.18 ; 4.69)$ \\
\hline Urinary metabolites & & $0.62(0.73 ; 4.35)$ & 0.079 \\
$\beta$-Alanine, ng/ $\mu \mathrm{g}$ creatinine & $1.44(0.61 ; 6.63)$ & $0.54(0.31 ; 0.89)$ & 0.38 \\
L-Proline, ng/ $\mu \mathrm{g}$ creatinine & $0.61(0.32 ; 0.93)$ & $21.0(12.2 ; 43.6)$ & 0.073 \\
Threonic acid, ng/ $\mu \mathrm{g}$ creatinine & $19.7(12.9 ; 40.0)$ & $120(78.3 ; 185)$ & 0.17 \\
1-Methylhistidine, ng/ $\mu \mathrm{g}$ creatinine & $108(71.3 ; 183)$ & $0.87(0.31 ; 13.0)$ & 0.002 \\
Malonic acid, ng/ $\mu \mathrm{g}$ creatinine & $1.33(0.31 ; 11.8)$ & $0.28(0.09 ; 0.77)$ & \\
Isovalerylcarnitine, ng/ $\mu \mathrm{g}$ creatinine & $0.17(0.05 ; 0.60)$ & & \\
\hline
\end{tabular}

Values are arithmetic mean \pm standard deviation, geometric mean ( 5 th and 95 th percentile), or estimated marginal mean (95\% confidence interval). * Adjusted for mean arterial pressure.

followed by a $7^{\circ} \mathrm{C} / \mathrm{min}$ increase to $120^{\circ} \mathrm{C}$. The oven temperature was then increased to $230^{\circ} \mathrm{C}$ at $10^{\circ} \mathrm{C} / \mathrm{min}$ and finally to $300^{\circ} \mathrm{C}$ at a rate of $13^{\circ} \mathrm{C} / \mathrm{min}$, where it was kept for $1 \mathrm{~min}$ before cooling to the initial temperature. The transfer line was kept constant at $225^{\circ} \mathrm{C}$ while the source was kept at $200^{\circ} \mathrm{C}$. Electron impact ionization $(-70 \mathrm{~V})$ was performed to fragment all eluting compounds. An acquisition delay of $230 \mathrm{~s}$ was allowed where after data were acquired at 20 spectra/s (50-950 m/z). The GC-TOF-MS data were processed with ChromaTOF (Leco) software. A baseline offset of 1 (just above the noise) was used with the "spanning" baseline tracking method to remove the baseline. The software performed auto smoothing. An average peak width of $3 \mathrm{~s}$ was selected to detect peaks with N20 signal-to-noise ratio and 5 apexing masses. The National Institute of Standards and Technology (2011) and an in-house-created library were used to identify the compound peaks through spectral matching.

All the samples were randomly assigned to be analysed in one of three batches on each of the different analytical platforms. The data were examined for within-batch and between-batch effects/drifts using quality control samples. Since no drifts were observed the data were processed further with standard metabolomics procedures. Briefly, a zero and quality control coefficient of variance filter was applied. The data were normalized (relatively quantified) by using the appropriate internal standards to eliminate any technical variance.

\section{Statistical Analysis}

By using the $G^{*}$ power v3.1.9.2 software, a priori power analysis showed that a population size of 72 participants (or 36 participants per group) was adequate for the hypothesis of this study [13]. Statistical analyses were performed with IBM ${ }^{\circledR}$ SPSS ${ }^{\circledR}$ Statistics, Version 24 software (IBM Corporation, Armonk, NY, USA). All variables were tested for normality by visual inspection (Q-Q plots) and evaluating skewness and kurtosis coefficients. In the case of a non-Gaussian distribution, a logarithmic transformation was performed for each skewed variable. All urinary metabolomics data were logarithmically transformed to obtain comparable concentration ranges of metabolites. Independent $t$ tests were used to compare the mean values (with standard deviations and 95\% confidence intervals) between groups. Analysis of covariance was used to adjust pulse wave velocity for mean arterial pressure. $\chi^{2}$ tests were used to compare proportions of dichotomous variables. Pearson and partial (adjusted for mean arterial pressure) correlations were performed to determine the links of arterial stiffness with urinary metabolites. Multiple regression analysis was performed 
Table 2. Unadjusted and adjusted correlations of pulse wave velocity with urinary metabolites in black and white boys

\begin{tabular}{|c|c|c|c|c|}
\hline & \multicolumn{4}{|c|}{ Pulse wave velocity, $\mathrm{m} / \mathrm{s}$} \\
\hline & \multicolumn{2}{|c|}{ Black children $(n=40)$} & \multicolumn{2}{|c|}{ White children $(n=40)$} \\
\hline & $r$ & $p$ & $r$ & $p$ \\
\hline$\beta$-Alanine, $\mathrm{ng} / \mu \mathrm{g}$ creatinine & -0.447 & 0.004 & -0.074 & 0.651 \\
\hline L-Proline, $\mathrm{ng} / \mu \mathrm{g}$ creatinine & 0.412 & 0.008 & -0.043 & 0.790 \\
\hline Threonic acid, ng/ $\mu$ g creatinine & 0.375 & 0.017 & -0.234 & 0.146 \\
\hline 1-Methylhistidine, $\mathrm{ng} / \mu \mathrm{g}$ creatinine & -0.336 & 0.034 & 0.058 & 0.724 \\
\hline Malonic acid, ng/ $\mathrm{gg}$ creatinine & 0.314 & 0.049 & 0.153 & 0.347 \\
\hline Isovalerylcarnitine, $\mathrm{ng} / \mu \mathrm{g}$ creatinine & -0.285 & 0.075 & -0.093 & 0.566 \\
\hline \multicolumn{5}{|c|}{ Partially adjusted for mean arterial pressure } \\
\hline$\beta$-Alanine, $\mathrm{ng} / \mu \mathrm{g}$ creatinine & -0.444 & 0.005 & -0.060 & 0.72 \\
\hline L-Proline, ng/ $\mu$ g creatinine & 0.417 & 0.008 & 0.037 & 0.82 \\
\hline 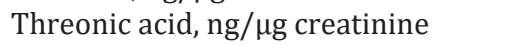 & 0.370 & 0.021 & -0.264 & 0.10 \\
\hline 1-Methylhistidine, $\mathrm{ng} / \mu \mathrm{g}$ creatinine & -0.353 & 0.027 & 0.082 & 0.62 \\
\hline Malonic acid, ng/ $\mathrm{gg}$ creatinine & 0.311 & 0.054 & 0.131 & 0.43 \\
\hline Isovalerylcarnitine, $\mathrm{ng} / \mu \mathrm{g}$ creatinine & -0.341 & 0.034 & -0.047 & 0.78 \\
\hline
\end{tabular}

to determine the independent relationships of pulse wave velocity with urinary metabolites: $\beta$-alanine, L-proline, 1-methylhistidine, malonic acid, threonic acid, and isovalerylcarnitine. Due to the small sample size, the covariates considered for entry to these models were limited to the three main contributors of pulse wave velocity: age, mean arterial pressure, and weight-to-height ratio.

\section{Results}

The general characteristics of the study population are described in Table 1. Age along with body composition variables were similar between black and white boys, except for weight-to-height ratio which was lower in the black compared to the white boys ( $p \leq 0.001)$. Pulse wave velocity, diastolic blood pressure and mean arterial pressure were higher in the black compared to the white boys (all $p \leq 0.002$ ). When comparing urinary metabolites between black and white boys, isovalerylcarnitine was lower in the black compared to the white boys ( $p=0.002)$. L-Proline levels were borderline higher $(p=0.079)$ and 1-methylhistidine levels borderline lower $(p=0.073)$ in the black compared to the white boys.

In both single and partial regression analyses (Table 2), pulse wave velocity correlated inversely with $\beta$-alanine and 1-methylhistidine $(p \leq 0.034)$ and positively with L-proline, threonic acid, and malonic acid in the black compared to white boys $(p \leq 0.054)$. In single regression analysis, pulse wave velocity tended to correlate inversely with isovalerylcarnitine in the black boys only; however, after adjusting for mean arterial pressure, this correlation became significant $(p=0.034)$.

In multiple regression analysis, the associations of pulse wave velocity with urinary metabolites were plotted in Figure 1. The associations demonstrated in single and partial regression analyses were confirmed in multiple regression analysis in the black boys only. Pulse wave velocity was associated inversely with $\beta$-alanine $(\beta=-0.414 ; p=0.008), 1$-methylhistidine $(\beta=-0.347 ; p=0.032)$, and isovalerylcarnitine $(\beta=-0.366 ; p=0.025)$ and positively with L-proline $(\beta=0.420 ; p=0.008)$, threonic acid $(\beta=0.977 ; p=0.033)$, and malonic acid $(\beta=0.348 ; p=0.030)$ in the black boys only. These associations were independent of age, weight-to-height ratio, and mean arterial pressure. 


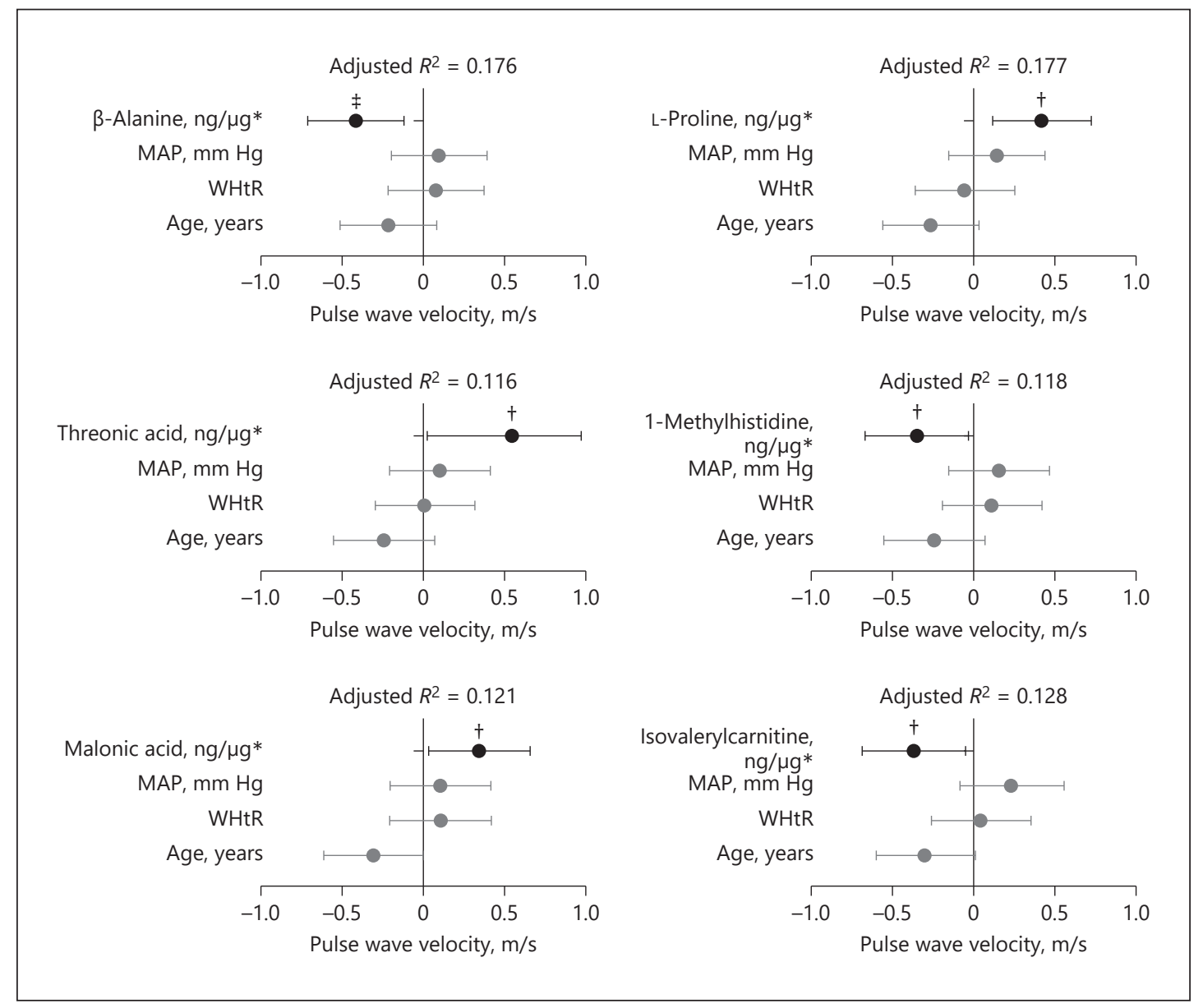

Fig. 1. Multiple regression analysis of pulse wave velocity with urinary metabolites in black boys. MAP, mean arterial pressure; WHtR, waist-to-height ratio. ${ }^{*}$ Units are expressed as ng/ $\mu$ g creatinine. ${ }^{\dagger} p \leq 0.05,{ }^{\ddagger} p<$ 0.001 .

\section{Discussion}

In this study we aimed to compare the urinary metabolite phenotypes between black and white boys and to explore associations of pulse wave velocity with particular urinary metabolites. We indicated that pulse wave velocity was positively associated with L-proline, threonic acid, and malonic acid and inversely with $\beta$-alanine, 1-methylhistidine, and isovalerylcarnitine in black boys only.

Studies implicated $\beta$-alanine, L-proline, and 1-methylhistidine in cardiac protection [14$16]$. $\beta$-Alanine has received a great deal of attention as an additional supplement for cardiac protection [15]. However, it appeared that the benefits of $\beta$-alanine were mostly due to one of its derivatives, carnosine [15]. Liver cells synthesize $\beta$-alanine after which it is transported in the plasma to where carnosine is required [16]. Carnosine is produced in the cardiac tissue and is directly dependent on its precursors, $\beta$-alanine and L-histidine, in which $\beta$-alanine is the rate-limiting factor [15]. Carnosine exerts antioxidant, anti-aging, procontractile, and membrane-protecting properties, and therefore also has anti-ischemic effects $[15,17]$. We therefore hypothesize that the inverse correlation of pulse wave velocity with $\beta$-alanine may indicate a potential reduced availability of the cardiac protective carnosine and could be 


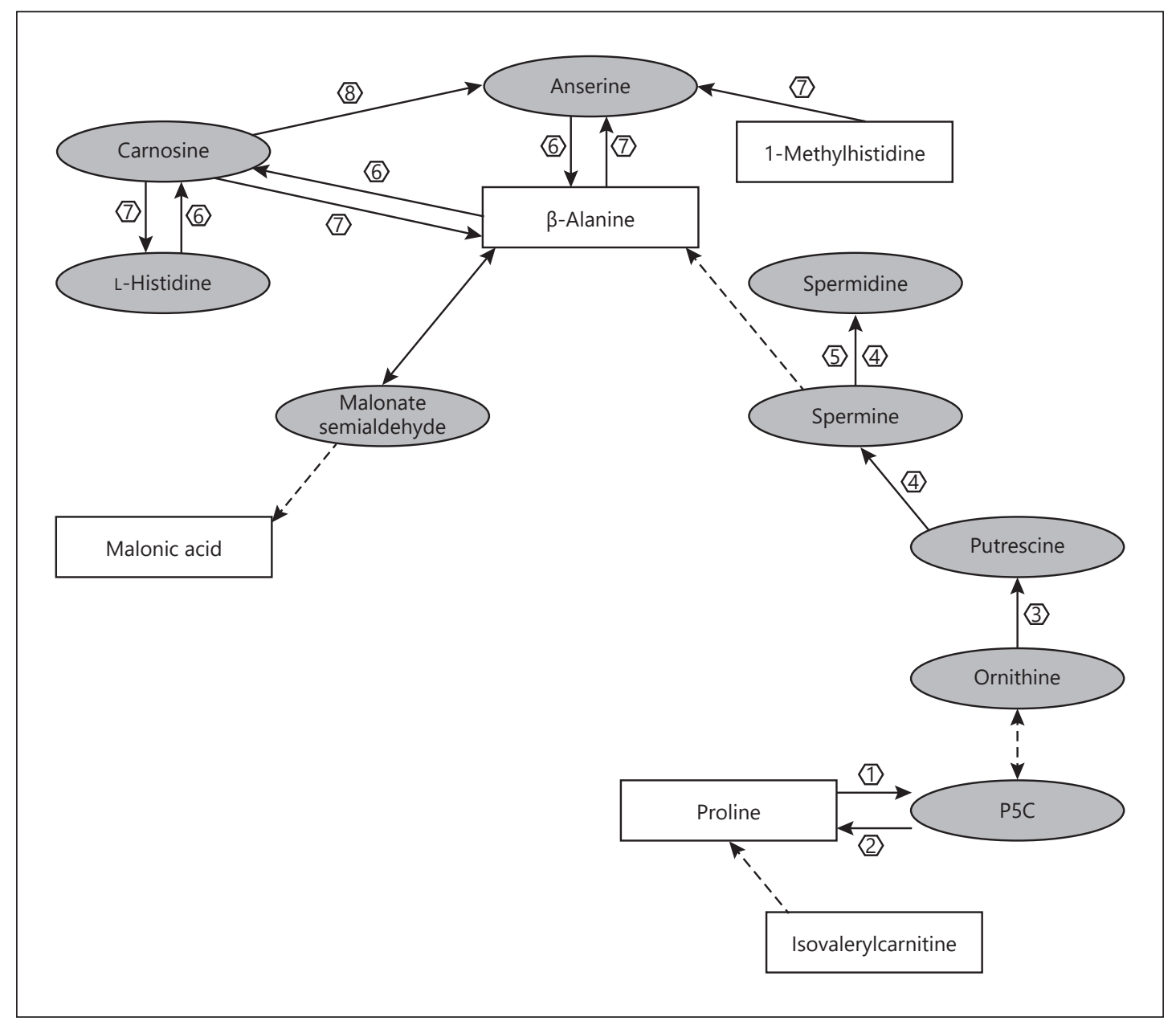

Fig. 2. Urinary metabolite scheme derived from KEGG pathways. The numbers denote the following enzymes: (1) proline dehydrogenase, (2) pyrroline-5-carboxylic acid (P5C) decarboxylase, (3) ornithine decarboxylase, (4) spermidine synthase, (5) spermine synthase, (6) carnosine synthase, (7) carnosine dipeptidase, (8) carnosine N-methyltransferase 1. Dashed arrows denote metabolites and enzymes not shown in the scheme.

partly involved in the early onset of arterial stiffness in this group of black boys. Although $\beta$-alanine levels did not differ between the boys, it is possible that even at the same $\beta$-alanine levels, a diminished cardiac protective effect of its derivative, carnosine, already exists. Since carnosine has various important cardiac protective functions, a reduced availability thereof may translate to a decrease in the exertion of its role as a cardiac protective agent and may therefore be associated with increased arterial stiffness in the black boys. Carnosine also regulates calcium concentrations in the sarcoplasmic reticulum $[15,17,18]$ and may therefore also, at reduced levels, impact the contractility of the heart. Reduced contractility of the heart may in turn impact adversely on arterial stiffness.

$\beta$-Alanine can further be metabolized to form anserine that can degenerate to $\beta$-alanine and 1-methylhistidine (Fig. 2). The 1-methylhistidine can further be metabolized to form anserine, an important free radical scavenger [19]. We consequently hypothesized that the inverse association of pulse wave velocity with 1-methylhistidine may indicate that a decrease in 1-methylhistidine may consequently lead to a decreased formation of anserine, with conse- 
quent increased oxidative stress, where the link between arterial stiffness and oxidative stress has previously been established [20]. Carnosine and anserine, the highest antioxidant in meat, is therefore an important dietary component [21], as previous results indicated 1-methylhistidine to be a urinary marker for meat consumption [22]. Previous studies indicated reduced dietary meat intake in black populations [5, 23], and it may therefore be plausible that the black boys of our study may also have decreased carnosine and anserine intake with possible adverse health consequences. However, this study lacked dietary intake data to support this hypothesis, and this should therefore be investigated in future studies.

Pulse wave velocity also associated positively with L-proline in the black boys. L-Proline is used as an alternative energy source under conditions of nutrient stress [24]. Black children may conceivably have a diminished balanced diet, which may contribute to unfulfilled nutrient needs [5, 23], causing L-proline to be used by the body as an alternative energy source. Studies done on L-proline pathways have linked L-proline as well as its derivatives (putrescine, spermine, and spermidine which are intermediates in the pathway between proline and $\beta$-alanine) with cardiac protective properties (Fig. 2) [25, 26]. L-Proline is a fundamental substrate in collagen and plays an important role in stimulating the biosynthesis thereof [27]. L-Proline is also stored as collagen in the extracellular matrix and connective tissue, such as in the vessels of the vascular system $[24,25]$, and is therefore important for stabilizing the vascular wall to have a sufficient collagen structure [16]. We therefore hypothesize that even though sufficient L-proline levels are necessary to maintain a stabilized vascular wall, if L-proline levels is elevated, it may lead to increased collagen biosynthesis and therefore decrease the elasticity of the extracellular matrix, contributing to increased arterial stiffness. Furthermore, L-proline exerts functions such as contributing to antioxidant activities, regulating cell differentiation, and playing a role in cell metabolism [24, 25, 27], and has also been shown to increase under cellular oxidative stress [25]. L-Proline is also connected to $\beta$-alanine metabolism as the precursor for ornithine, which is the precursor for putrescine, the latter being the precursor for spermine $[25,28,29]$. We therefore hypothesize that the borderline higher L-proline levels in the black boys may be increased to account for the decrease in other antioxidants such as $\beta$-alanine and carnosine. We also speculate that the pathway leading from L-proline to $\beta$-alanine may be compromised, therefore leading to increased L-proline levels and decreased $\beta$-alanine. Further studies are encouraged to confirm this hypothesis. Polyamines such as putrescine, spermine, and spermidine, which are intermediates in the pathway between L-proline and $\beta$-alanine, show important functions in regulating cell growth, cell functions, and cell differentiation [30,31]. Spermidine and putrescine inhibit platelet aggregation and repair arteries due to vascular injuries [32]. Spermidine supplementation studies on mice showed reduced reactive oxygen species and collagen deposition, restored endothelial dependent nitric oxide, and reversed arterial aging [26]. It is consequently probable that L-proline levels are upregulated to produce more spermidine and spermine to counteract increased arterial stiffness with its cardiac protective effects, as seen with our positive association between pulse wave velocity and L-proline.

Isovalerylcarnitine (C5 acylcarnitine) is related to branched chain amino acid catabolism $[33,34]$. Studies have shown that branched chain amino acids such as leucine, isoleucine, and valine, as well as C5-acylcarnitines were linked with CVD and the development thereof [33, 34]. However, since no associations of pulse wave velocity with valine or leucine/isoleucine were indicated in the exploratory phase of this study (data not shown), this association should be interpreted with caution. Less is known about the association of pulse wave velocity with malonic and threonic acid and more research is needed to explore this further.

All urinary metabolites where measured by using state-of-the-art analytical techniques such as the LC-MS/MS and GC-TOF-MS methods. Although our study consisted of a small population group, a priori power analysis showed that a total population size of 72 partici- 
pants was adequate for the hypothesis of this study. Our study is one of the first studies to make use of cardiovascular measurements with metabolomics and to contribute to the limited data available on arterial stiffness with metabolomics in black and white boys from South Africa. Even though our study consists of hypothesis-generating research, the importance thereof of contributing to hypothesis-testing research should not be underestimated. These identified metabolites may be possible CVD risk markers and future studies may prove these markers to be important targets in the prevention and prediction of CVD development, and therefore decrease the increasing tendency of CVD.

In conclusion, we showed possible adverse contributions of $\beta$-alanine, 1-methylhistidine, and L-proline to arterial stiffness in black boys at the young age of 6-8 years. These associations were completely independent of major contributing factors to pulse wave velocity such as age, blood pressure, and body composition.

\section{Disclosure Statement}

The research funded in this paper is part of the Arterial Stiffness in Offspring Study (ASOS) which was financially supported by the National Research Foundation (NRF) of South Africa, in part supported by the Extramural Research Unit of the South African Medical Research Council (SAMRC) hosted at the North-West University within the Hypertension in Africa Research Team (HART), and by industry funding from the South African Sugar Association (SASA). Any opinion, findings, and conclusions or recommendations expressed in this material are those of the authors, and therefore, the NRF does not accept any liability in this regard. The authors have no conflict of interest to declare.

\section{References}

1 Yusuf S, Lonn E, Pais P, Bosch J, López-Jaramillo P, Zhu J, et al: Blood-pressure and cholesterol lowering in persons without cardiovascular disease. N Engl J Med 2016;374:2032-2043.

2 Mosca L, Benjamin E, Berra K, Bezanson J, Dolor R, Lloyd-Jones D, et al: Effectiveness-based guidelines for the prevention of cardiovascular disease in women - 2011 update: a guideline from the American Heart Association. Circulation 2011;123:1-20.

3 Afshin A, Penalvo J, Del Gobbo L, Kashaf M, Micha R, Morrish K, et al: CVD prevention through policy: a review of mass media, food/menu labeling, taxation/subsidies, built environment, school procurement, worksite wellness, and marketing standards to improve diet. Curr Cardiol Rep 2015;17:98.

4 Mokwatsi GG, Schutte AE, Kruger R: Ethnic differences regarding arterial stiffness of 6-8-year-old black and white boys. J Hypertens 2017;35:960-967.

5 Schutte AE, Huisman HW, Van Rooyen JM, De Ridder JH, Malan NT: Associations between arterial compliance and anthropometry of children from four ethnic groups in South Africa: the THUSA BANA Study. Blood Press 2003;12:97-103.

6 Olsen MH, Angell SY, Asma S, Boutouyrie P, Burger D, Chirinos JA, et al: A call to action and a lifecourse strategy to address the global burden of raised blood pressure on current and future generations: the Lancet Commission on hypertension. Lancet 2016;388:2665-2712.

7 Djekic D, Nicoll R, Novo M, Henein M: Metabolomics in atherosclerosis. IJC Metab Endocr 2015;8:26-30.

8 Lewis GD, Asnani A, Gerszten RE: Application of metabolomics to cardiovascular biomarker and pathway discovery. J Am Coll Cardiol 2008;52:117-123.

9 Dunn WB, Erban A, Weber RJ, Creek DJ, Brown M, Breitling R, et al: Mass appeal: metabolite identification in mass spectrometry-focused untargeted metabolomics. Metabolomics 2013;9:44-66.

10 Bouatra S, Aziat F, Mandal R, Guo AC, Wilson MR, Knox C, et al: The human urine metabolome. PLoS One 2013; 8:e73076.

11 Stewart A M-JM, Olds T, De Ridder H: International Standards for Anthropometric Assessment. Lower Hutt, ISAK, 2011.

12 Van Bortel LM, Laurent S, Boutouyrie P, Chowienczyk P, Cruickshank JK, De Backer T, et al: Expert consensus document on the measurement of aortic stiffness in daily practice using carotid-femoral pulse wave velocity. J Hypertens 2012;30:445-448.

13 Faul F, Erdfelder E, Lang A-G, Buchner A: G*Power 3: A flexible statistical power analysis program for the social, behavioral, and biomedical sciences. Behav Res Methods 2007;39:175-191.

14 Wu H-C, Shiau C-Y, Chen H-M, Chiou T: Antioxidant activities of carnosine, anserine, some free amino acids and their combination. J Food Drug Anal 2003;11:148-153. 
Erasmus et al.: Metabolomics and Arterial Stiffness in Boys

15 McCarty MF, DiNicolantonio JJ: $\beta$-Alanine and orotate as supplements for cardiac protection. Open Heart 2014; 1:e000119.

16 Ivanov V, Roomi MW, Kalinovsky T, Niedzwiecki A, Rath M: Anti-atherogenic effects of a mixture of ascorbic acid, lysine, proline, arginine, cysteine, and green tea phenolics in human aortic smooth muscle cells. J Cardiovasc Pharmacol 2007;49:140-145.

17 Stvolinsky S, Dobrota D: Anti-ischemic activity of carnosine. Biochemistry (Mosc) 2000;65:849-855.

18 Culbertson JY, Kreider RB, Greenwood M, Cooke M: Effects of beta-alanine on muscle carnosine and exercise performance: a review of the current literature. Nutrients 2010;2:75-98.

19 Fu H, Katsumura Y, Lin M, Muroya Y, Hata K, Fujii K, et al: Free radical scavenging and radioprotective effects of carnosine and anserine. Radiat Phys Chem 2009;78:1192-1197.

20 Mokhaneli MC, Fourie CMT, Botha S, Mels CMC: The association of oxidative stress with arterial compliance and vascular resistance in a bi-ethnic population: the SABPA study. Free Radic Res 2016;50:920-928.

21 Williams P: Nutritional composition of red meat. Nutr Diet 2007;64:S113-S119.

22 Myint T, Fraser GE, Lindsted KD, Knutsen SF, Hubbard RW, Bennett HW: Urinary 1-methylhistidine is a marker of meat consumption in Black and in White California Seventh-day Adventists. Am J Epidemiol 2000;152: 752-755.

23 MacIntyre U, Kruger H, Venter C, Vorster H: Dietary intakes of an African population in different stages of transition in the North West Province, South Africa: the THUSA study. Nutr Res 2002;22:239-256.

24 Phang JM, Pandhare J, Liu Y: The metabolism of proline as microenvironmental stress substrate. J Nutr 2008; 138:2008S-2015S.

25 Wu G, Bazer FW, Burghardt RC, Johnson GA, Kim SW, Knabe DA, et al: Proline and hydroxyproline metabolism: implications for animal and human nutrition. Amino Acids 2011;40:1053-1063.

26 LaRocca TJ, Gioscia-Ryan RA, Hearon CM, Seals DR: The autophagy enhancer spermidine reverses arterial aging. Mech Ageing Dev 2013;134:314-320.

27 Karna E, Miltyk W, Wołczyński S, Pałka JA: The potential mechanism for glutamine-induced collagen biosynthesis in cultured human skin fibroblasts. Comp Biochem Physiol B Biochem Mol Biol 2001;130:23-32.

28 Madeo F, Eisenberg T, Büttner S, Ruckenstuhl C, Kroemer G: Spermidine: a novel autophagy inducer and longevity elixir. Autophagy 2010;6:160-162.

29 Kanehisa M, Furumichi M, Tanabe M, Sato Y, Morishima K: KEGG: new perspectives on genomes, pathways, diseases and drugs. Nucleic Acids Res 2017;45(D1):D353-D361.

30 Zhao Y-J, Xu C-Q, Zhang W-H, Zhang L, Bian S-L, Huang Q, et al: Role of polyamines in myocardial ischemia/ reperfusion injury and their interactions with nitric oxide. Eur J Pharmacol 2007;562:236-246.

31 Moinard C, Cynober L, de Bandt J-P: Polyamines: metabolism and implications in human diseases. Clin Nutr 2005;24:184-197.

32 Martin-Lorenzo M, Zubiri I, Maroto AS, Gonzalez-Calero L, Posada-Ayala M, de la Cuesta F, et al: KLK1 and ZG16B proteins and arginine-proline metabolism identified as novel targets to monitor atherosclerosis, acute coronary syndrome and recovery. Metabolomics 2015;11:2.

33 Shah SH, Bain JR, Muehlbauer MJ, Stevens RD, Crosslin DR, Haynes C, et al: Association of a peripheral blood metabolic profile with coronary artery disease and risk of subsequent cardiovascular events. Circ Cardiovasc Genet 2010;3:207-214.

34 Batch BC, Shah SH, Newgard CB, Turer CB, Haynes C, Bain JR, et al: Branched chain amino acids are novel biomarkers for discrimination of metabolic wellness. Metabolism 2013;62:961-969. 